\title{
Virtual monoenergetic images from spectral detector computed tomography facilitate washout assessment in arterially hyper-enhancing liver lesions
}

\author{
R. P. Reimer ${ }^{1}$ (D) N. Große Hokamp ${ }^{1}$ • A. Fehrmann Efferoth ${ }^{1} \cdot$ A. Krauskopf $^{2}$ • D. Zopfs ${ }^{1} \cdot$ J. R. Kröger ${ }^{1} \cdot$ T. Persigehl $^{1}$. \\ D. Maintz ${ }^{1}$ - A. C. Bunck ${ }^{1}$
}

Received: 27 March 2020 / Revised: 20 August 2020 / Accepted: 6 October 2020 / Published online: 12 November 2020

(C) The Author(s) 2020

\begin{abstract}
Objectives To investigate whether the increased soft tissue contrast of virtual monoenergetic images (VMIs) obtained from a spectral detector computed tomography (SDCT) system improves washout assessment of arterially hyper-enhancing liver lesions. Methods Fifty-nine arterially hyper-enhancing lesions in 31 patients (age $65 \pm 9$ years, M/W 20/11) were included in this IRBapproved study. All patients underwent multi-phase SDCT for HCC screening. MRI, CEUS or biopsy within 3 months served as standard of reference to classify lesions as LiRADS 3 or 4/5. VMIs and conventional images (CIs) were reconstructed. Visual analysis was performed on 40,60 , and 80 kiloelectronvolt $(\mathrm{keV})$ and CIs by 3 radiologists. Presence and visibility of washout were assessed; image quality and confidence of washout evaluation were evaluated on 5-point Likert scales. Signal-to-noise ratio (SNR), lesion-to-liver contrast-to-noise ratio (CNR) $\left(\mid \mathrm{HU}_{\text {lesion }}-\mathrm{HU}_{\text {liver }} / \mathrm{SD}_{\text {liver }}\right)$ and washout $\left(\mid \mathrm{HU}_{\text {lesion }}-\mathrm{HU}_{\text {liver }}\right)$ were calculated. Statistical assessment was performed using ANOVA and Wilcoxon test.

Results On subjective lesion analysis, the highest level of diagnostic confidence and highest sensitivity for the detection of lesion washout were found for $40-\mathrm{keV}$ VMIs $(40 \mathrm{keV}$ vs. CI, $81.3 \mathrm{vs}$. $71.3 \%)$. Image quality parameters were significantly better in lowkiloelectronvolt VMIs than in CIs ( $p<0.05$; e.g. SNR liver: $40 \mathrm{keV}$ vs. CIs, $12.5 \pm 4.1$ vs. $5.6 \pm 1.6)$. In LiRADS $4 / 5$ lesions, CNR and quantitative washout values were significantly higher in $40-\mathrm{keV}$ VMIs compared to CIs $(p<0.05$; e.g. CNR and washout in $40 \mathrm{keV}$ vs. CIs, $2.3 \pm 1.6$ vs. $0.8 \pm 0.5$ and $29.0 \pm 19.1$ vs. $12.9 \pm 6.9 \mathrm{HU}$, respectively).

Conclusion By increasing lesion contrast, low-kiloelectronvolt VMIs obtained from SDCT improve washout assessment of hyper-enhancing liver lesions with respect to washout visibility and diagnostic confidence.

Key Points

- Low-kiloelectronvolt virtual monoenergetic images from spectral detector CT facilitate washout assessment in arterially hyperenhancing liver lesions.

- Image quality and quantitative washout parameters as well as subjective washout visibility and diagnostic confidence benefit from low-kiloelectronvolt virtual monoenergetic images.
\end{abstract}

Keywords Carcinoma, hepatocellular · Tomography, X-ray computed $\cdot$ Diagnostic imaging

Electronic supplementary material The online version of this article (https://doi.org/10.1007/s00330-020-07379-3) contains supplementary material, which is available to authorized users.

R. P. Reimer robert.reimer@uk-koeln.de

1 Department of Diagnostic and Interventional Radiology, Faculty of Medicine and University Hospital Cologne, University of Cologne, Kerpener Str. 62, 50937 Cologne, Germany

2 Department of Diagnostic and Interventional Radiology, University Düsseldorf, Medical Faculty, 40225 Düsseldorf, Germany

$\begin{array}{ll}\text { Abbreviations } \\ \text { CE } & \text { Contrast-enhanced } \\ \text { CIs } & \text { Conventional images } \\ \text { DECT } & \text { Dual-energy CT } \\ \text { HCC } & \text { Hepatocellular carcinoma } \\ \text { keV } & \text { Kiloelectronvolt } \\ \text { SDCT } & \text { Spectral detector CT } \\ \text { VMIs } & \text { Virtual monoenergetic images }\end{array}$




\section{Introduction}

Hepatocellular carcinoma (HCC) may be diagnosed by contrast-enhanced (CE) magnetic resonance imaging (MRI), computed tomography (CT), ultrasound (CEUS) or a combination of these modalities without the need for a histological confirmation by biopsy $[1,2]$. Many studies and metaanalyses investigated and compared the diagnostic performances of the different modalities with the predominant result that MRI provides the highest accuracy, especially in small liver lesions [2-4]. Although, these studies slightly favour MRI over CT, both are equally recommended as modality of choice for the diagnostic evaluation of patients at risk of HCC, due to their high sensitivity and coverage allowing a comprehensive assessment of the entire liver [1, 2]. However, MRI may be contraindicated in some patients or may result in insufficient image quality, e.g. in patients with ascites or in patients incapable of holding their breath. Furthermore, diagnosis may require a confirmation by a second imaging modality if imaging features are equivocal or a lesion has been assigned an intermediate probability for malignancy $[2,5]$.

Typical radiological findings in HCC include a combination of an arterial hyper-enhancement (APHE) with nonperipheral washout in portal-venous and/or delayed phase in CT/MRI or late washout ( $>60 \mathrm{~s}$ ) in CEUS, enhancing capsule and/or lesion threshold growth on a follow-up scan $[2,5,6]$. The assessment of washout, defined as a hypointensity, hypo-density or hypoechogenicity compared to the adjacent liver parenchyma, can be difficult in cases with only subtle differences [7].

In the past decade, dual-energy CT (DECT) raised interest in the field of liver imaging, since it has been shown to improve soft tissue contrast by means of virtual monoenergetic images (VMIs). DECT systems register low- and high-energy data attenuation profiles enabling a reconstruction of VMIs, which approximate images from an acquisition with a true monoenergetic X-ray beam [8-10].

While different emission-based DECT systems have been available for more than a decade, recently, a detector-based approach was introduced, referred to as spectral detector CT (SDCT). By using a dual-layer detector, it enables a simultaneous detection of low- and high-energy photons with complete temporal and spatial registration, allowing for a retrospective reconstruction of VMIs in a range from 40 to 200 kiloelectronvolt $(\mathrm{keV})$ for every scan [10-12].

VMIs at low kiloelectronvolts are known to improve soft tissue contrast due to the energy dependence of the linear attenuation coefficients while maintaining low image noise throughout the available $\mathrm{keV}$ range [10]. Furthermore, multiple studies reported an increase in detectability and conspicuity of arterially hyper-enhancing liver lesions in VMIs using low-kiloelectronvolt values [10,13-15]. Additional studies showed better visualization of hypodense liver lesions, while only few focused on HCC [14-18].
The aim of our study was to analyse whether the increased soft tissue contrast by means of VMIs improves the washout assessment of arterially hyper-enhancing liver lesions in contrast-enhanced SDCT.

\section{Material and methods}

\section{Study population}

This retrospective study was conducted in compliance with the protocol and the principles laid down in the Declaration of Helsinki, in accordance with the ICH Harmonized Tripartite Guideline for Good Clinical Practice. The institutional review board waived informed consent. A structured search in the radiology information system was performed with the following inclusion criteria: (1) older than 18 years of age, (2) multi-phase contrast-enhanced SDCT for HCC workup between May 2016 and November 2018, (3) examination with a standardized imaging protocol as described below, (4) arterially hyper-enhancing liver lesions of $1 \mathrm{~cm}$ or greater and (5) MRI, CEUS or biopsy within 3 months. A maximum of 5 lesions per patient was included.

Exclusion criteria comprised (1) prior locoregional therapy and (2) modified imaging protocol and incomplete image reconstructions. Imaging studies were reviewed using a clinical DICOM-Viewer (Impax EE R20, Dedalus Group). Contrast-enhanced liver MRI, CEUS or biopsy within 3 months served as reference standard to categorize lesions according to the current LiRADS classification system $[5,6]$. All biopsy-positive lesions were rated as LiRADs 5 lesions. In total, 31 patients were identified exhibiting a total of 59 arterially hyper-enhancing focal liver lesions (ESM1).

\section{Acquisition parameters}

All CT scans were performed for clinical indications on the same spectral detector CT scanner (IQon Spectral CT, Philips Healthcare). Patients were scanned in cranio-caudal direction and a head-first, supine position. Our institution's HCC workup protocol for CT examinations contains an unenhanced, arterial, portal-venous and delayed scan of the liver, while the portal-venous scan covers the abdomen and pelvis, with or without a chest scan. The arterial, portal-venous and delayed phases were acquired using an automated bolustracking technique (threshold of $150 \mathrm{HU}$ in the abdominal aorta) with a delay of 15,50 and $240 \mathrm{~s}$, respectively. Administration of $100 \mathrm{ml}$ non-ionic, iodinated contrast media bolus (Accupaque $350 \mathrm{mg} / \mathrm{ml}$, GE Healthcare) followed by a $30-\mathrm{ml}$ saline chaser is routinely performed with an automated injection system at a flow rate of $4.0 \mathrm{ml} / \mathrm{s}$ (MEDRAD ${ }^{\circledR}$ Stellant, Bayer Vital GmbH). Tube current modulation was 
Table 1 Radiation dose

\begin{tabular}{llccc}
\hline CT phase & Rotation time $(\mathrm{s})$ & Tube current-time product $(\mathrm{mAs})$ & DLP $(\mathrm{mGy} \times \mathrm{cm})$ & CTDI $_{\mathrm{vol}}(\mathrm{mGy})$ \\
\hline Unenhanced & 0.33 & $143 \pm 46.7$ & $342.1 \pm 130.0$ & $13.0 \pm 4.2$ \\
Arterial & 0.5 & $131.5 \pm 50.6$ & $307.9 \pm 136.6$ & $12.0 \pm 4.6$ \\
Portal-venous & 0.33 & $144.1 \pm 52.0$ & $871.1 \pm 335.7$ & $13.1 \pm 4.7$ \\
Delayed & 0.33 & $131.8 \pm 50.7$ & $309.2 \pm 137.3$ & $12.0 \pm 4.6$ \\
\hline
\end{tabular}

$D L P$, dose-length product; $C T D I_{v o l}$, volumetric CT dose index. Results are means \pm standard deviation

activated in all patients (DoseRight 3D-DOM, Philips Healthcare). Scan parameters were as follows: collimation $64 \times 0.625 \mathrm{~mm}$, tube voltage $120 \mathrm{kVp}$, pitch 0.485 . In portal-venous scans covering the chest and abdomen, pitch was 0.671 (Table 1).

\section{Image reconstruction}

Image reconstruction for further analysis was limited to portalvenous and delayed phases only. Conventional images (CIs) were reconstructed using a hybrid iterative reconstruction algorithm (iDose ${ }^{4}$, Philips Healthcare) with a standard soft tissue kernel (B). VMIs of 40-200 keV with a $10-\mathrm{keV}$ increment were reconstructed using a dedicated, hybrid iterative spectral reconstruction algorithm (Spectral, Kernel B, Philips Healthcare). In SDCT, information from each layer is used to generate photoelectric-like and Compton-like images, which are then linearly blended to generate VMIs. Although the detailed reconstruction process remains undisclosed by the vendor, it appears that some sort of noise reduction is used [19]. Denoising for both was set to a medium level (level 3 of 7). All images were reconstructed with a slice thickness of $2 \mathrm{~mm}$ and a section increment of $1 \mathrm{~mm}$.

\section{Qualitative analysis}

All 59 included lesions were qualitatively analysed by one board-certified body radiologist (13 years of experience in liver imaging) and two diagnostic radiology residents with 1 -year experience in body CT. Analysis was divided into two parts with an interval of 3 months in between to avoid recall bias. For the analysis, reading sessions were prepared by one of the study team members not involved in the blinded reading. Qualitative assessment was limited to CIs and three representative VMI energy levels $(40,60$ and $80 \mathrm{keV})$ of portal-venous and delayed phases, while arterial phase was only used to indicate lesions to be evaluated. The three readers were free to adjust window settings and scroll through the lesions.

In the first part of our analysis, prepared reading sessions displayed the arterial phase with the lesion of interest encircled on the left screen and the portal-venous and delayed phases with the same image reconstruction on the right screen. First, presence of washout was rated (yes/no) for portal-venous and delayed phases, respectively. Second, if washout was present in both phases, readers were asked to decide which phase displayed washout better. Furthermore, overall image quality and diagnostic confidence of washout evaluation were rated on 5-point Likert scales, being adapted from previous studies $[14,17,20]$ : for overall image quality, ranging from 1 , nondiagnostic, to 5, extraordinary; and for diagnostic confidence of washout evaluation, ranging from 1 , none, to 5 , extraordinary (ESM2).

As washout was only or better seen on delayed-phase images in $72.2 \%$ in the first part of the qualitative reading, the second part of the qualitative analysis as well as the quantitative analysis was limited to the delayed phase only. For the second part of the qualitative analysis, prepared reading sessions displayed the arterial phase with the arterially hyperenhancing lesion of interest encircled on the left screen and all blinded delayed-phase image reconstructions $(40,60$, $80 \mathrm{keV}$ and CIs) in a randomized order next to each other on the right screen. In this reading, readers were asked to choose the image reconstruction with the best washout to liver contrast, hereafter referred to as best washout visibility.

For statistical analysis, washout was only considered present, if confidence was rated moderate or higher, since otherwise the diagnosis probably would have not been made in clinical routine.

\section{Quantitative analysis}

Quantitative analysis was performed using region-of-interest (ROI)-based measurements of mean HU and standard deviation (SD). Circular ROIs were placed in the following structures: 2 in lesions, 2 in adjacent liver parenchyma, 1 in autochthonous back musculature and 1 in portal vein. All ROIs were drawn as large as possible (at least $0.5 \mathrm{~cm}^{2}$ ) and were only adjusted to exclude unrepresentative structures such as vessels, bile ducts, areas of focal liver cirrhosis and fasciae. In heterogeneous lesions, ROIs were placed in areas that visually appeared most hypodense. ROIs were placed on CIs and copied to identical positions in all reconstructed VMIs. Mean and $\mathrm{SD}$ of HU were averaged where applicable. Image noise was represented by $\mathrm{SD}$ in back musculature. Signal-to-noise ratio $\left(\mathrm{SNR}_{x}\right)$ of a $\mathrm{ROI}_{\mathrm{x}}$ was defined as $\mathrm{SNR}_{x}=\mathrm{HU}_{x} / \mathrm{SD}_{x}$, lesion-toliver contrast-to-noise ratio $(\mathrm{CNR})$ as $\mathrm{CNR}=\mid \mathrm{HU}_{\text {lesion }}{ }^{-}$ 
$\mathrm{HU}_{\text {liver }} / \mathrm{SD}_{\text {liver }}$ being adapted from previous studies $[10,14]$ and quantitative washout values as $\left|\mathrm{HU}_{\text {lesion }}-\mathrm{HU}_{\text {liver }}\right|$.

\section{Statistical analysis}

All analyses were performed using JMP Software (Version 14, SAS Institute $\mathrm{GmbH}$ ) unless specified below. To compare groups, we used ANOVA or Wilcoxon tests, adjusted for multiple comparisons if appropriate. A $p$ value $<0.05$ was considered significant. Continuous variables are reported as mean $\pm \mathrm{SD}$ and Likert scores as median (quartiles). Sensitivities and specificities are given with corresponding 95\% confidence intervals. Inter-rater reliability was determined by means of intra-class correlation estimates (ICCs) using RStudio (Version 1.1.456; RStudio) based on a mean of 3 raters, consistency and 2-way mixed-effects model for the qualitative analysis [21]. Inter-rater agreement was evaluated as described earlier [22].

\section{Results}

\section{Study cohort}

The mean age of patients was $65 \pm 9$ years; of these patients, $12(35.5 \%)$ were women and $20(64.5 \%)$ men (ESM1). In total, 59 arterially hyper-enhancing lesions were assessed. According to the reference standards, lesions were classified as LiRADS $3(n=9), 4(n=2)$ and $5(n=48)$ (Table 2). Mean lesion size was $3.0 \pm 2.4 \mathrm{~cm}$ (range, $1.0-13.0 \mathrm{~cm}$ ).

Table 2 LiRADS classification according to the reference standard

\begin{tabular}{llll}
\hline & LiRADS 3 & LiRADS 4 & LiRADS 5 \\
\hline MRI, no washout & $8(13.6)$ & - & - \\
MRI, no APHE; washout $(<2 \mathrm{~cm})$ & $1(1.7)$ & & \\
MRI, capsule & - & $2(3.4)$ & - \\
MRI, washout & - & - & $16(27.1)$ \\
CEUS, washout & - & - & $3(5.1)$ \\
Biopsy, HCC & - & - & $21(35.6)$ \\
MRI and CEUS, washout & - & - & $2(3.4)$ \\
MRI, no washout; CEUS, washout & - & - & $1(1.7)$ \\
MRI, no washout; & - & - & $1(1.7)$ \\
Biopsy, HCC & & & $3(5.1)$ \\
MRI, washout; Biopsy, HCC & - & - & $1(1.7)$ \\
CEUS, washout; Biopsy, HCC & - & - & \\
\hline
\end{tabular}

LiRADS, Liver Imaging Reporting and Data System; MRI, magnetic resonance imaging; APHE, Arterial phase hyper-enhancement; CEUS, contrast-enhanced ultrasound; $H C C$, hepatocellular carcinoma. Results are lesion number (\%)

\section{Qualitative analysis}

The overall intraclass correlation between the 3 independent readers was $0.897(0.883-0.909)$, indicating an excellent interreader reliability. Regarding washout evaluation, the overall ICC was 0.915 (0.895-0.933), in $40 \mathrm{keV} 0.857$ $(0.780-0.910)$, in $60 \mathrm{keV} 0.958(0.936-0.974)$, in $80 \mathrm{keV}$ VMIs $0.904(0.852-0.940)$ and in CIs $0.936(0.901-0.960)$, and regarding confidence of washout evaluation 0.707 (0.635-0.766), 0.789 (0.674-0.867), 0.669 (0.490-0.793), $0.565(0.330-0.727)$ and $0.781(0.662-0.862)$, respectively.

Overall image quality was rated best in $60-\mathrm{keV}$ VMIs, while $60-\mathrm{keV}$ VMIs and $80-\mathrm{keV}$ VMIs were rated significantly better compared to CIs (both $p<0.05$ ); 40-keV VMIs were rated slightly better compared to CIs without reaching statistical significance $(p>0.05)$ (Table 3$)$.

The confidence of washout evaluation was rated in descending order from $40-\mathrm{keV}, 60-\mathrm{keV}$ and $80-\mathrm{keV}$ VMIs to CIs, only reaching statistical significance when comparing $40-\mathrm{keV}$ images and CIs $(p<0.05)$ (Table 3).

Six arterially hyper-enhancing lesions exhibited no washout on any of the imaging modalities. In the remaining 53 lesions, washout was identified on CT images by at least one reader (Fig. 1). As mentioned above, washout was only $(32.3 \%)$ or better $(39.9 \%)$ seen on delayed-phase CT images in $72.2 \%$, while it was better seen in portal-venous phase in $27.8 \%$.

The diagnostic sensitivity to detect washout in LiRADS 4/5 lesions was highest in 40-keV VMIs (81.3\% (73.8-86.5\%)) followed by $60-\mathrm{keV}(78.2 \%$ (70.9-84.1\%)) and $80-\mathrm{keV}$ VMIs (75.5\% (68.0-81.8\%)) and CIs (72.1\% (64.4-78.7\%)). Specificity was highest in CIs $(96.3 \%$ (81.7-99.3\%) followed by $60-\mathrm{keV}$ (92.6\% (76.6-97.9\%), 80-keV (92.6\% (76.697.9\%) and 40-keV VMIs (88.9\% (71.9-96.1\%) (Table 3). Interestingly, 2 LIRADS 4 lesions with a capsule but no washout on MRI and 2 LIRADS 3 lesions without washout on MRI exhibited washout on SDCT (altogether 2 lesions on CIs, 2 lesions on $80-\mathrm{keV}, 3$ lesions on $60-\mathrm{keV}$ and 4 lesions on $40-$ $\mathrm{keV}$ VMIs). On direct comparison, 40-keV VMIs were chosen to show best washout visibility in $88.3 \%$, followed by 60 $\mathrm{keV}$ VMIs (5.1\%), CIs (4.4\%) and 80-keV VMIs (2.2\%) (Table 3).

\section{Quantitative analysis}

\section{Image quality parameters}

Lesions exhibited average HU of $75.4 \pm 29.0$ on CIs. For VMIs, a stepwise decrease of HU was found from low to high kiloelectronvolt. Compared to CIs and as expected, HU were significantly higher in VMIs with 40-60-keV levels and significantly lower in VMIs with $80-200 \mathrm{keV}(p<0.05$; Fig. 2, Table 4). Image noise was highest in CIs $(15.4 \pm 3.0 \mathrm{HU})$ and 
Table 3 Results of qualitative analysis

\begin{tabular}{lllll}
\hline & CIs & $40-\mathrm{keV}$ VMIs & $60-\mathrm{keV}$ VMIs & 80-keV VMIs \\
\hline $\begin{array}{l}\text { Image quality } \\
\text { Washout }\end{array}$ & $4(3-5)$ & $4(3-5)$ & $4(4-5)$ & $4(4-5)$ \\
Sensitivity, \% & $71.3(63.6-78.0)$ & $81.3(74.3-86.8)$ & $78.7(71.4-84.5)$ & $74.7(67.2-81.0)$ \\
Specificity, \% & $96.3(81.7-99.3)$ & $88.9(71.9-96.1)$ & $92.6(76.6-97.9)$ & $92.6(76.6-97.9)$ \\
Confidence & $4(3-5)$ & $5(4-5)$ & $5(4-5)$ & $4(3-5)$ \\
Best visible, \% & 4.4 & 88.3 & 5.1 & 2.2 \\
\hline
\end{tabular}

$C I s$, conventional images; VMIs, virtual monoenergetic images. Results are median (quartiles), unless specified significantly higher in 40-keV VMIs $(12.8 \pm 2.7 \mathrm{HU})$ compared to energy levels of $60-\mathrm{keV}(11.7 \pm 2.2 \mathrm{HU})$ and above $(p<0.05$; Fig. 2, Table 4). SNRs of lesions and liver were significantly highest in $40-\mathrm{keV}$ VMIs, respectively $(p<0.05)$. It decreased with increasing $\mathrm{keV}$ levels and was significantly higher in VMIs with 40-90-keV levels compared to CIs ( $p<0.05$; Fig. 2, Table 4$)$.

\section{Washout evaluation}

The following results compare LiRADS 3 and 4/5 lesions where applicable.

On equivalent image reconstructions, CNR and quantitative washout values were significantly higher in LiRADS 4/5 compared to LiRADS 3 lesions (e.g. CNR in CIs $0.8 \pm 0.5$ vs. $0.3 \pm 0.3$, and washout in $40 \mathrm{keV} 29.0 \pm 19.1$ vs. $15.1 \pm 15.8$ $\mathrm{HU} ; p<0.05)$.

In LiRADS 4/5 lesions, CNR and washout values decreased with increasing kiloelectronvolt levels. Both were significantly highest in 40-keV VMIs, while CNR was significantly higher in 40-70-keV levels compared to CIs and washout values were significantly higher in $40-$ and $50-\mathrm{keV}$ levels compared to CIs $(p<0.05$; Fig. 3, Table 5$)$.

Image examples are shown in Figs. 4 and 5.

\section{Discussion}

Our study confirms an improved washout assessment of arterially hyper-enhancing liver lesions for contrast-enhanced SDCT-derived low-kiloelectronvolt VMIs. Resulting from an increased soft tissue contrast, we observed best washout visibility, diagnostic sensitivity and confidence for $40-\mathrm{keV}$ VMIs compared to all other tested reconstructions. With respect to washout evaluation in particular, overall ICC was excellent among the three readers.

So far, only few studies exist on the added value of DECT for the diagnostic workup of HCC [13, 14, 17]. Moreover, to
Fig. 1 Venn diagram displaying the number of lesions with washout in any of the CT reconstructions diagnosed by any of the readers in comparison to the reference standards. Washout was diagnosed in all lesions with a positive reference standard, except for one lesion with a combination of a positive biopsy and washout in CEUS.

Furthermore, both LiRADS 4 lesions with a capsule in MRI and 2 LiRADS 3 lesions without washout in MRI were diagnosed with washout in CT

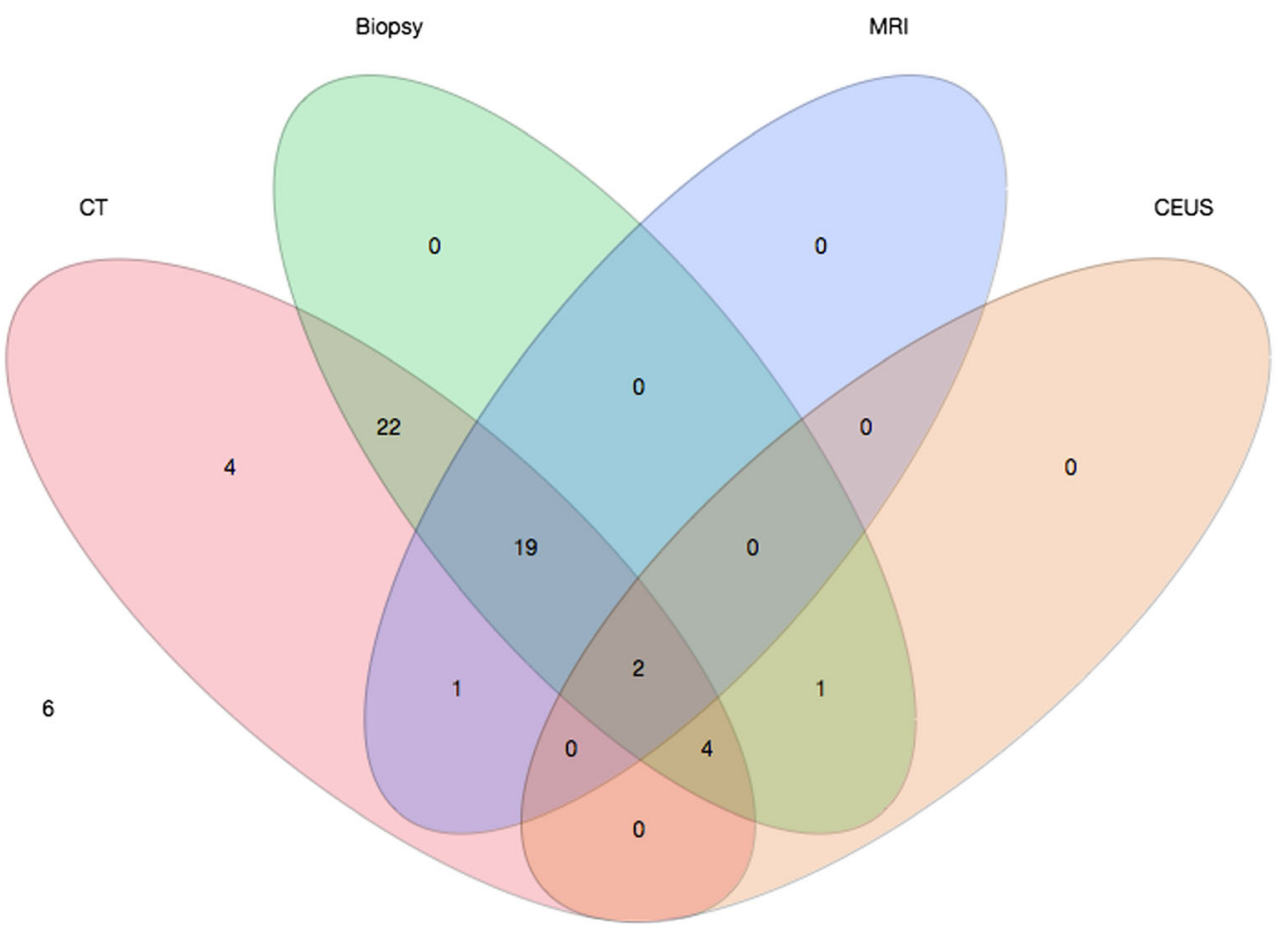


a

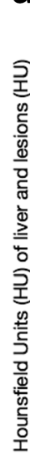

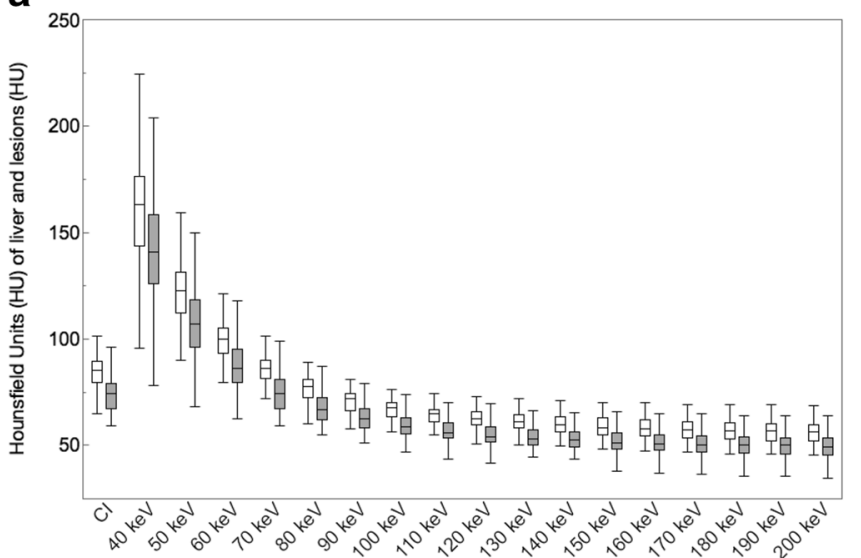

b

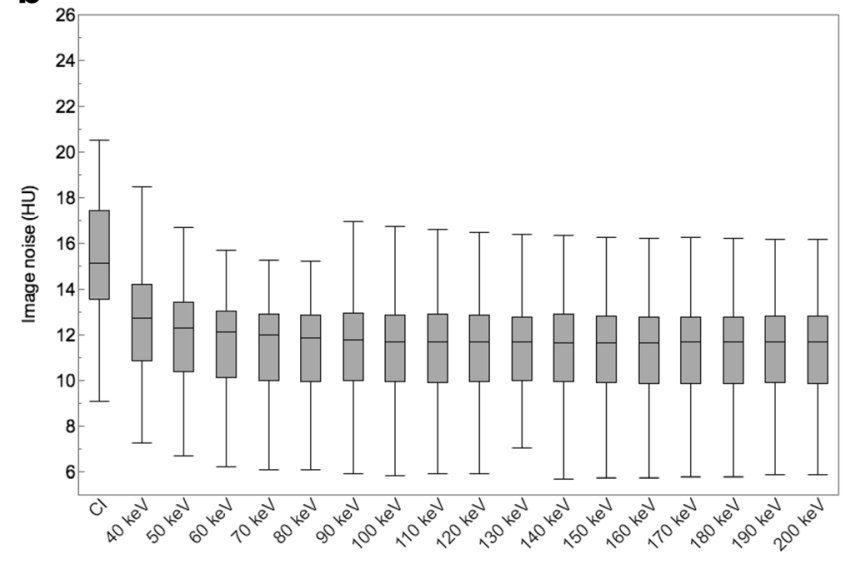

C

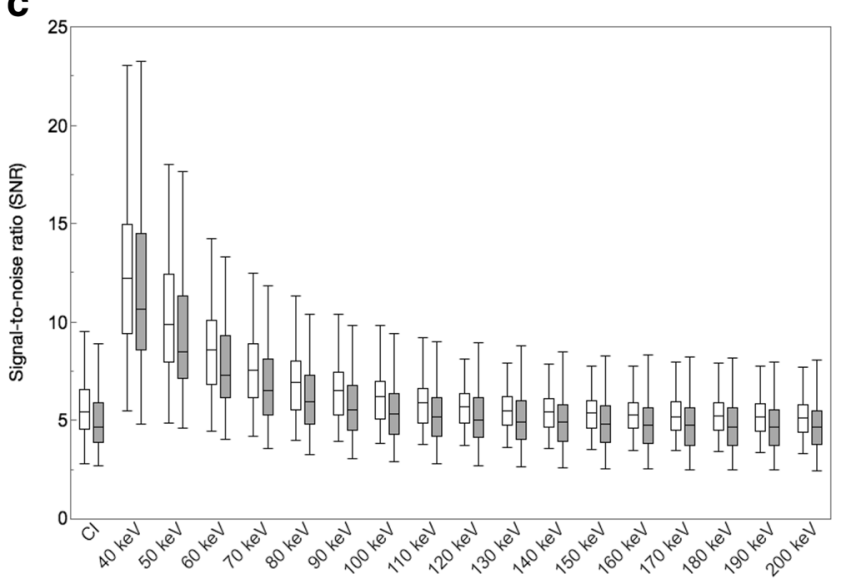

Fig. 2 Illustration of mean Hounsfield units (HU) of liver parenchyma (empty boxplots) and lesions (filled boxplots) clearly reveals higher HU in low-kiloelectronvolt virtual monoenergetic images (VMIs) compared to conventional images (CIs) (a). Noise, as depicted by standard deviation (SD) of the autochthonous back musculature, differed in dependency of

our knowledge, there is only a single report on the added value of dual-energy CT for visual washout assessment in HCC [17]. In their study, Matsuda et al assessed washout visibility on virtual monoenergetic reconstructions acquired during equilibrium phase. Matsuda's and our study differ in a number of aspects, in particular with respect to inclusion criteria
$\mathrm{keV}$ level in VMIs; however, it was found to be lower in any of the VMIs compared to CIs (b). This resulted in clear superiority of signal-to-noise ratio for liver (empty boxplots) and lesions (filled boxplots) in 40-90-keV images compared to CIs, respectively (c)

(lesions $>1 \mathrm{~cm}$ fulfilling HCC criteria on conventional CT images vs. any arterially hyper-enhancing lesions $>1 \mathrm{~cm}$ ), the injected contrast dose (adjusted to body surface area vs. fixed dose) and the timing of delayed-phase acquisition (100 s vs. $240 \mathrm{~s}$ after a trigger threshold of 100 and $150 \mathrm{HU}$, respectively) [17]. The longer delay chosen in our study is in line
Table 4 Quantitative results of image quality parameters

\begin{tabular}{lccccc}
\hline & CIs & 40-keV VMIs & $80-\mathrm{keV}$ VMIs & 120-keV VMIs & 200-keV VMIs \\
\hline $\begin{array}{l}\left.\text { Noise (SD } \mathrm{SD}_{\text {mscle }}\right) \\
\text { Lesion }\end{array}$ & $15.4 \pm 3.0$ & $12.8 \pm 2.7$ & $11.4 \pm 2.1$ & $11.3 \pm 2.1$ & $11.3 \pm 2.1$ \\
$\quad$ & & & & \\
Attenuation & $74.8 \pm 9.0$ & $140.6 \pm 25.4$ & $67.9 \pm 7.8$ & $55.7 \pm 6.8$ & $50.7 \pm 6.9$ \\
SNR & $5.0 \pm 1.6$ & $11.8 \pm 4.4$ & $6.3 \pm 1.9$ & $5.3 \pm 1.5$ & $4.8 \pm 1.4$ \\
Liver & & & & & \\
Attenuation & $84.3 \pm 8.9$ & $160.9 \pm 32.4$ & $76.4 \pm 6.6$ & $62.3 \pm 5.1$ & $56.4 \pm 5.5$ \\
SNR & $5.6 \pm 1.6$ & $12.5 \pm 4.1$ & $7.0 \pm 1.7$ & $5.8 \pm 1.4$ & $5.3 \pm 1.2$ \\
\hline
\end{tabular}

$C I s$, conventional images; VMIS, virtual monoenergetic images; $S D$, standard deviation; $S N R$, signal-to-noise ratio. Results are means \pm standard deviation 
a

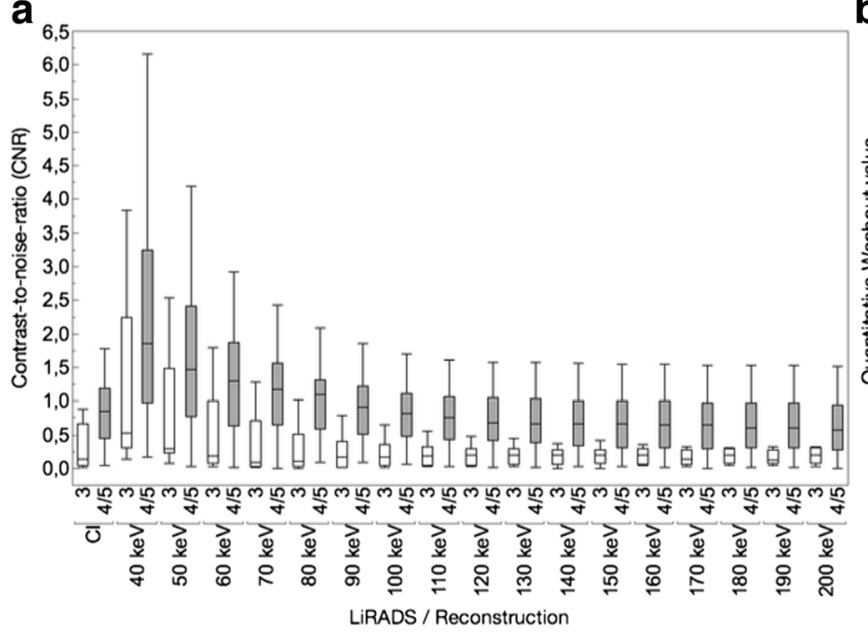

b

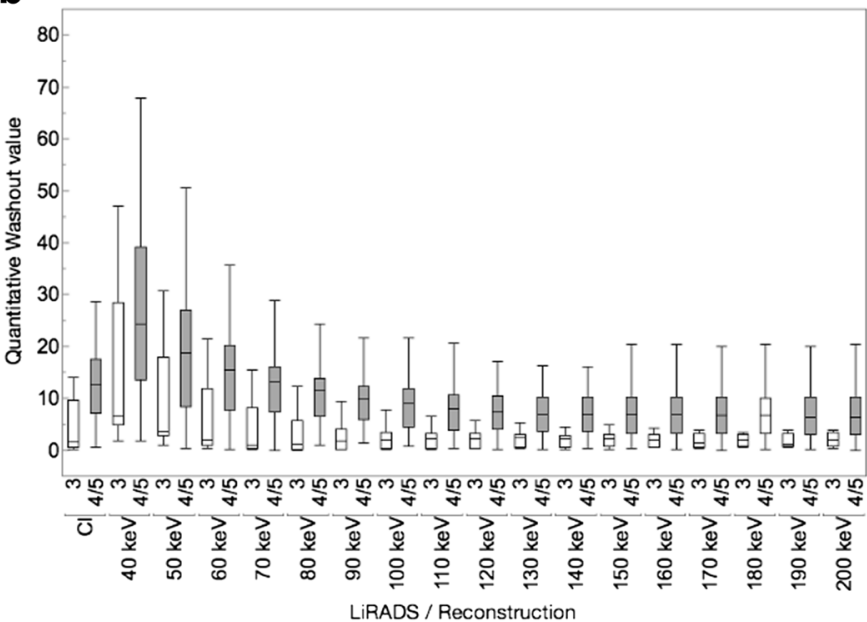

Fig. 3 Illustrations clearly show a higher lesion-to-liver contrast-to-noise ratio (CNR) and quantitative washout values in LiRADS 4/5 (filled boxplots) compared to LiRADS 3 lesions (empty boxplots) $(\mathbf{a}, \mathbf{b})$

with protocol recommendations by the American College of Radiology, which advise a delay of $2-5 \mathrm{~min}$ [23]. On delayedphase images, enhancement of liver parenchyma was well above the recommended $50 \mathrm{HU}$ in all of our reconstructions. Despite the outlined differences, Matsuda et al reported similar results with significantly higher SNR, CNR and washout values in noise-reduced low mono-kiloelectronvolt images [17]. The improved image quality of SDCT-derived lowkiloelectronvolt images observed in our study is in accordance with earlier studies [10, 16, 24].

By also including lesions with no apparent signs of washout on conventional CT reconstructions in our study, we were able to demonstrate that low-kiloelectronvolt VMIs not only improve diagnostic confidence but also may increase the sensitivity for the detection of washout of arterially enhancing lesion on delayed-phase images. Depending on the reader, the number of lesions that were confidently identified to exhibit some degree of washout increased with the assessment of 40-keV VMIs by 10-35\% compared to CIs. Moreover, all biopsy-proven lesions and 4 lesions with no signs of washout on MR imaging were identified to exhibit signs of washout on SDCT images. As a consequence, two LiRADS 3 and both LiRADS 4 lesions would have been upgraded to LiRADS 5 based on $40-$ and $60-\mathrm{keV}$ images. In light of these results, it was surprising that one LiRADS 5 lesion with a heterogeneous APHE and obvious signs of washout on delayedphase CIs and $80-\mathrm{keV}$ images partially exhibited hyperdense $\mathrm{HU}$ on $40-\mathrm{keV}$ images compared to the adjacent liver parenchyma hampering washout assessment in this case (Fig. 5). This case illustrates that VMIs should always be assessed in conjunction with CIs.

While a systematic comparison of the sensitivity of washout detection between portal-venous and delayed phases was not the main focus of our study, we observed a clear advantage for delayed-phase images with $72 \%$ of lesions exhibiting signs of washout only or better on delayed-phase images. This is in good agreement with several studies that have proven a clear benefit for delayed-phase images over portal-venous-phase images for the detection of lesion washout [25].

Other studies investigating HCC using DECT focussed on quantitative imaging parameters for decision-making [18, 26, 27]. For example, Pfeiffer et al proposed various imaging parameters using $\mathrm{HU}$ values and iodine concentration in and between arterial and portal-venous phase for the identification of HCC, while Laroia et al investigated iodine density of lesions in arterial phase for the assessment of $\operatorname{HCC}[18,26]$.
Table 5 Quantitative results of washout assessment

\begin{tabular}{lccccc}
\hline & CIs & 40-keV VMIs & 80-keV VMIs & 120-keV VMIs & 200-keV VMIs \\
\hline CNR & & & & & \\
LiRADS 3 & $0.3 \pm 0.3$ & $1.2 \pm 1.3$ & $0.3 \pm 0.4$ & $0.2 \pm 0.2$ & $0.2 \pm 0.1$ \\
LiRADS 4/5 & $0.8 \pm 0.5$ & $2.3 \pm 1.6$ & $1.0 \pm 0.5$ & $0.7 \pm 0.4$ & $0.6 \pm 0.4$ \\
Washout & & & & & \\
LiRADS 3 & $4.5 \pm 5.2$ & $15.1 \pm 15.8$ & $3.3 \pm 4.2$ & $2.1 \pm 1.8$ & $2.0 \pm 1.3$ \\
LiRADS 4/5 & $12.9 \pm 6.9$ & $29.0 \pm 19.1$ & $10.9 \pm 5.6$ & $8.2 \pm 5.0$ & $7.1 \pm 5.2$ \\
\hline
\end{tabular}

$C I s$, conventional images; VMIs, virtual monoenergetic images; $C N R$, contrast-to-noise ratio; LiRADS, Liver Imaging Reporting and Data System. Results are means \pm standard deviation 
Fig. 4 Axial CT images showing an arterially hyper-enhancing lesion in liver segment VII (a) with washout in delayed phase on CI (b), $40 \mathrm{keV}$ (c) and $60 \mathrm{keV}$ (d). However, washout and lesion-toliver ratio were higher in $40-\mathrm{keV}$ compared to $60-\mathrm{keV}$ images and CIs, resulting in a better visibility
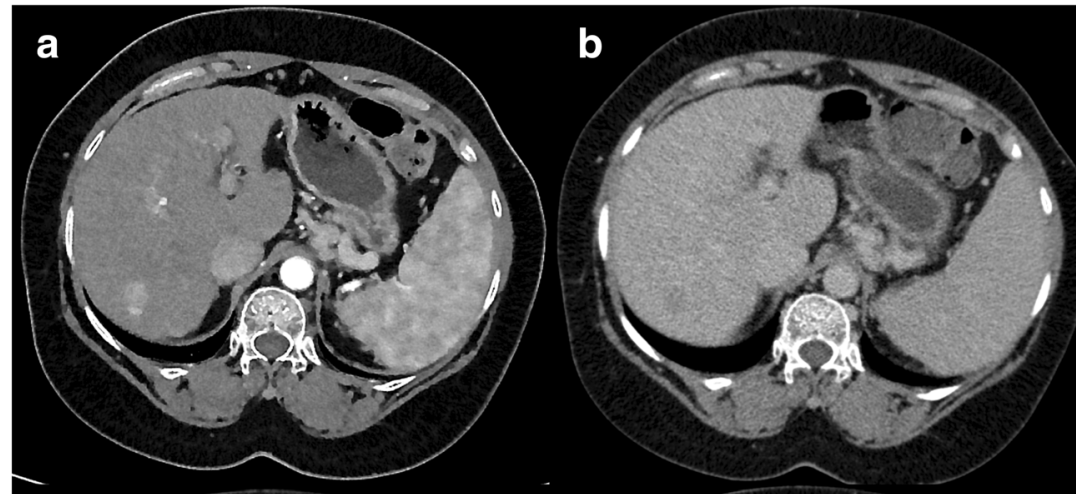

C

d
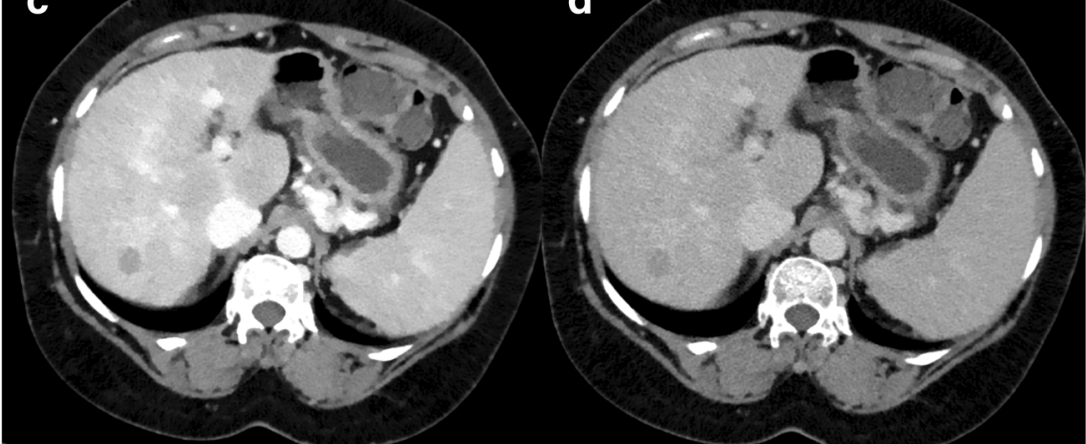

Another study by Liu et al using conventional CT scanners introduced a percentage-ratio approach for HCC detection using the arterial, portal-venous and delayed phases [27]. While our quantitative measurements confirm an improved CNR between lesions with washout and adjacent liver parenchyma for low-kiloelectronvolt VMIs, subjective analysis of
HCC remains the gold standard in clinical routine. This is especially true for heterogeneous lesions, in which only certain areas may exhibit signs of washout.

Besides the retrospective study design, several limitations need to be addressed. First, the study only includes a limited number of patients and lesions. Second, histological
Fig. 5 Axial CT images showing an arterially hyper-enhancing heterogeneous lesion in liver segment VII (a) with washout in delayed phase on CIs (b) and suggested on $60-\mathrm{keV}$ images (d). However, the lesion partially exhibited hyperdense Hounsfield units on 40-keV images compared to the liver parenchyma (c). The lesion also showed washout in the reference MRI and was categorized as LiRADS 5, which was proved by biopsy

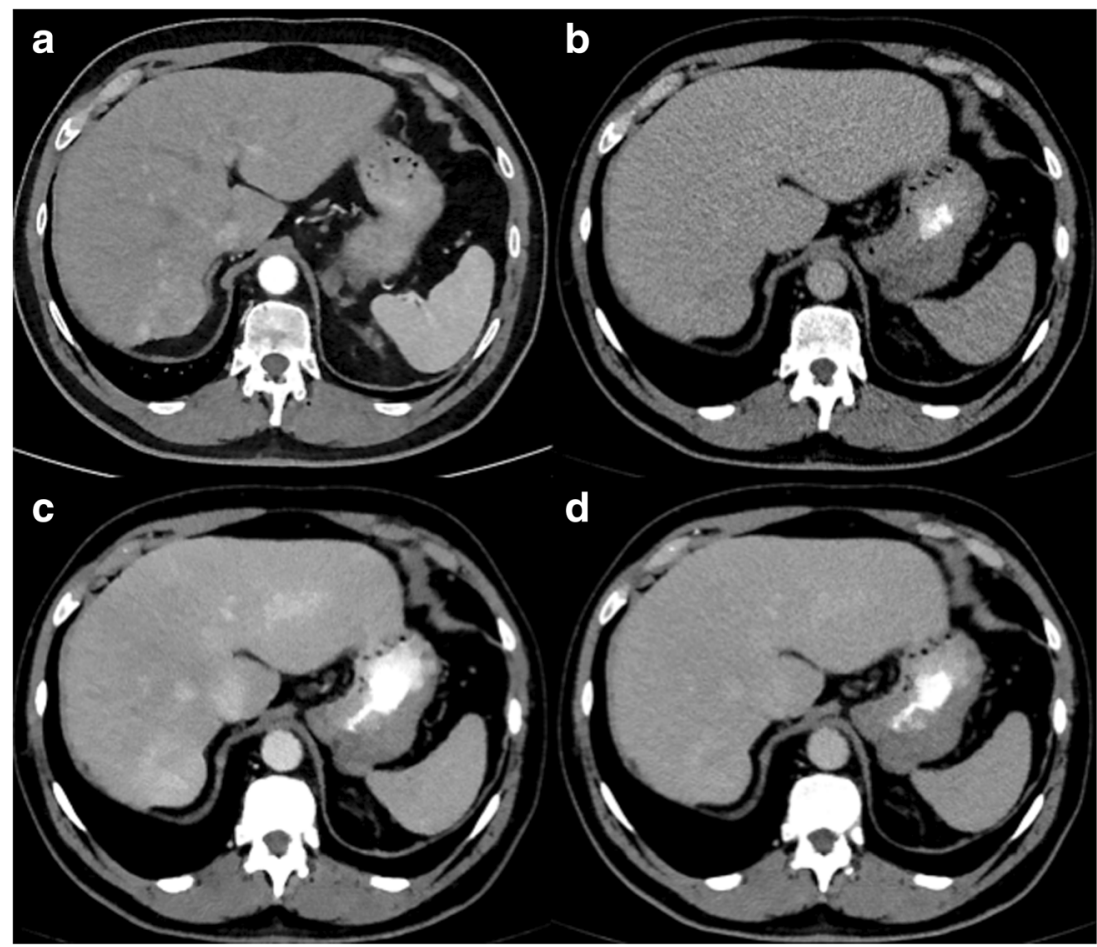


confirmation and grading was not available for all lesions, especially no large population of dysplastic nodules of various grading or early HCCs were included in this first study using SDCT. Also MRI was not available in all cases impeding a direct comparison of diagnostic performance between both modalities. As a consequence, estimates of diagnostic accuracy need to be interpreted with caution. In particular, the reduced specificity of $40-$ and $60-\mathrm{keV}$ images for washout detection should not be interpreted as an increased rate of false positives, but is more likely to be explained by the limited sensitivity of our reference imaging modalities. Fourth, the impact of different-sized ROIs on our results was not assessed. Furthermore, it must be emphasized that conventional CT images remain the gold standard, while VMIs may be beneficial to be additionally reviewed in uncertain situations. Last, we quantitatively and qualitatively assessed washout of known arterially hyper-enhancing lesions in this proof of concept study; however, based on these results, a further large-scale study to investigate the value of SDCT in comparison to CT, MRI and CEUS for non-invasive detection and characterization of liver lesions in high-risk patients is desired. Its exact impact on the diagnostic accuracy in comparison to the other modalities remains to be determined.

In conclusion, virtual monoenergetic images obtained with spectral detector CT using low-kiloelectronvolt images may improve the assessment of washout in arterially hyper-enhancing liver lesions. Hence, these reconstructions seem beneficial in uncertain situations to improve confidence of washout evaluation and visibility in delayed-phase images.

Funding Open Access funding enabled and organized by Projekt DEAL.

\section{Compliance with ethical standards}

Guarantor The scientific guarantor of this study is Alexander Christian Bunck.

Conflict of interest David Maintz and Nils Große Hokamp received speaker's honoraria from Philips Healthcare. Nils Große Hokamp receives research support from Philips Healthcare.

Statistics and biometry No complex statistical methods were necessary for this paper.

Informed consent Written informed consent was waived by the Institutional Review Board.

Ethical approval Institutional Review Board Approval was obtained.

\footnotetext{
Methodology

- retrospective

- diagnostic or prognostic study

- performed at one institution
}

Open Access This article is licensed under a Creative Commons Attribution 4.0 International License, which permits use, sharing, adaptation, distribution and reproduction in any medium or format, as long as you give appropriate credit to the original author(s) and the source, provide a link to the Creative Commons licence, and indicate if changes were made. The images or other third party material in this article are included in the article's Creative Commons licence, unless indicated otherwise in a credit line to the material. If material is not included in the article's Creative Commons licence and your intended use is not permitted by statutory regulation or exceeds the permitted use, you will need to obtain permission directly from the copyright holder. To view a copy of this licence, visit http://creativecommons.org/licenses/by/4.0/.

\section{References}

1. Marrero JA, Kulik LM, Sirlin CB et al (2018) Diagnosis, staging, and management of hepatocellular carcinoma: 2018 Practice Guidance by the American Association for the Study of Liver Diseases. Hepatology 68:723-750. https://doi.org/10.1002/hep. 29913

2. Galle PR, Forner A, Llovet JM et al (2018) EASL Clinical Practice Guidelines: Management of hepatocellular carcinoma. J Hepatol 69:182-236. https://doi.org/10.1016/j.jhep.2018.03.019

3. Li J, Wang J, Lei L et al (2019) The diagnostic performance of gadoxetic acid disodium-enhanced magnetic resonance imaging and contrast-enhanced multi-detector computed tomography in detecting hepatocellular carcinoma: a meta-analysis of eight prospective studies. Eur Radiol 29:6519-6528. https://doi.org/10.1007/ s00330-019-06294-6

4. Hanna RF, Miloushev VZ, Tang A et al (2016) Comparative 13year meta-analysis of the sensitivity and positive predictive value of ultrasound, CT, and MRI for detecting hepatocellular carcinoma. Abdom Radiol (NY) 41:71-90. https://doi.org/10.1007/s00261015-0592-8

5. American College of Radiology: Liver Imaging Reporting and Data System (2018) CT/MRI LI-RADS® v2018. https://www.acr.org/ Clinical-Resources/Reporting-and-Data-Systems/LI-RADS/CTMRI-LI-RADS-v2018. Accessed 20 Jan 2019

6. Dietrich CF, Fetzer D, Jang H-J et al (2017) CEUS LI-RADS ® v2017 CORE. In: Am. Coll. Radiol. https://www.acr.org/ClinicalResources/Reporting-and-Data-Systems/LI-RADS/CEUS-LIRADS-v2017. Accessed 8 Oct 2019

7. Navin PJ, Venkatesh SK (2019) Hepatocellular carcinoma: state of the art imaging and recent advances. J Clin Transl Hepatol 7:1-14. https://doi.org/10.14218/jcth.2018.00032

8. Alvarez RE, Macovski A (1976) Energy-selective reconstructions in X-ray computerized tomography. Phys Med Biol 21:733-744. https://doi.org/10.1088/0031-9155/21/5/002

9. Neuhaus V, Abdullayev N, Große Hokamp N et al (2017) Improvement of image quality in unenhanced dual-layer CT of the head using virtual monoenergetic images compared with polyenergetic single-energy CT. Invest Radiol 52:470-476. https://doi.org/10.1097/RLI.0000000000000367

10. Große Hokamp N, Höink AJ, Doerner J et al (2018) Assessment of arterially hyper-enhancing liver lesions using virtual monoenergetic images from spectral detector CT: phantom and patient experience. Abdom Radiol (NY) 43:2066-2074. https://doi.org/10.1007/ s00261-017-1411-1

11. McCollough CH, Leng S, Yu L, Fletcher JG (2015) Dual- and multi-energy CT: principles, technical approaches, and clinical applications. Radiology 276:637-653. https://doi.org/10.1148/radiol. 2015142631 
12. Lennartz S, Laukamp KR, Neuhaus V et al (2018) Dual-layer detector CT of the head: initial experience in visualization of intracranial hemorrhage and hypodense brain lesions using virtual monoenergetic images. Eur J Radiol 108:177-183. https://doi.org/ 10.1016/j.ejrad.2018.09.010

13. Shuman WP, Green DE, Busey JM et al (2014) Dual-energy liver CT: effect of monochromatic imaging on lesion detection, conspicuity, and contrast-to-noise ratio of hypervascular lesions on late arterial phase. AJR Am J Roentgenol 203:601-606. https://doi.org/ 10.2214/AJR.13.11337

14. Lv P, Lin XZ, Chen K, Gao J (2012) Spectral CT in patients with small HCC: investigation of image quality and diagnostic accuracy. Eur Radiol 22:2117-2124. https://doi.org/10.1007/s00330-0122485-3

15. Husarik DB, Gordic S, Desbiolles L et al (2015) Advanced virtual monoenergetic computed tomography of hyperattenuating and hypoattenuating liver lesions: ex-vivo and patient experience in various body sizes. Invest Radiol 50:695-702. https://doi.org/10. 1097/RLI.0000000000000171

16. Große Hokamp N, Obmann VC, Kessner R et al (2018) Improved visualization of hypodense liver lesions in virtual monoenergetic images from spectral detector CT: proof of concept in a 3Dprinted phantom and evaluation in 74 patients. Eur J Radiol 109: 114-123. https://doi.org/10.1016/j.ejrad.2018.11.001

17. Matsuda M, Tsuda T, Kido T et al (2018) Dual-energy computed tomography in patients with small hepatocellular carcinoma: utility of noise-reduced monoenergetic images for the evaluation of washout and image quality in the equilibrium phase. J Comput Assist Tomogr 42:937-943. https://doi.org/10.1097/RCT.0000000000000752

18. Laroia ST, Bhadoria A, Venigalla Y et al (2016) Role of dual energy spectral computed tomography in characterization of hepatocellular carcinoma: initial experience from a tertiary liver care institute. Eur J Radiol Open 3:162-171. https://doi.org/10.1016/j. ejro.2016.05.007

19. Hokamp NG, Maintz D, Shapira N et al (2020) Technical background of a novel detector-based approach to dual-energy computed tomography. Diagn Interv Radiol 26:68-71. https://doi.org/10. 5152/dir.2019.19136
20. Zopfs D, Laukamp KR, Pinto dos Santos D et al (2019) Low-keV virtual monoenergetic imaging reconstructions of excretory phase spectral dual-energy CT in patients with urothelial carcinoma: a feasibility study. Eur J Radiol 116:135-143. https://doi.org/10. 1016/j.ejrad.2019.05.003

21. Gamer M, Lemon J, Fellows I, Singh P (2012) Package irr: various coefficients of interrater reliability and agreement. Available via https://cran.r-project.org/web/packages/irr/irr.pdf. Accessed 25 Mar 2019

22. Cohen J (1960) A coefficient of agreement for nominal scales. Educ Psychol Meas XX:37-46

23. Kambadakone A, Chandarana H, Chernyak V et al (2018) LIRADS ® Technique. Am Coll Radiol. Available via https:/www. acr.org/-/media/ACR/Files/Clinical-Resources/LIRADS/Chapter12 - $\mathrm{T}$ e c h n i q u e . p d f ? 1 a $=$ e $\mathrm{n} \& \mathrm{~h}$ a $\mathrm{s} \mathrm{h}=$ 3B774BD8A6D0A6ACBD62B2330705FD14. Accessed 12 Mar 2019

24. Große Hokamp N, Obmann VC, Kessner R et al (2018) Virtual monoenergetic images for diagnostic assessment of hypodense lesions within the liver: semiautomatic estimation of window settings using linear models. J Comput Assist Tomogr 42:925-931. https:// doi.org/10.1097/RCT.0000000000000794

25. Monzawa S, Ichikawa T, Nakajima H et al (2007) Dynamic CT for detecting small hepatocellular carcinoma: usefulness of delayed phase imaging. AJR Am J Roentgenol 188:147-153. https://doi. org/10.2214/AJR.05.0512

26. Pfeiffer D, Parakh A, Patino M et al (2018) Iodine material density images in dual-energy CT: quantification of contrast uptake and washout in HCC. Abdom Radiol (NY) 43:3317-3323. https://doi. org/10.1007/s00261-018-1636-7

27. Liu YI, Shin LK, Jeffrey RB, Kamaya A (2013) Quantitatively defining washout in hepatocellular carcinoma. AJR Am J Roentgenol 200:84-89. https://doi.org/10.2214/AJR.11.7171

Publisher's note Springer Nature remains neutral with regard to jurisdictional claims in published maps and institutional affiliations. 